\title{
Performance of Multi-chaotic PSO on a Shifted Benchmark Functions Set
}

\author{
Michal Pluhacek, Roman Senkerik and Ivan Zelinka \\ Tomas Bata University in Zlin, Faculty of Applied Informatics \\ Department of Informatics and Artificial Intelligence \\ nám. T.G. Masaryka 5555, 76001 Zlín, Czech Republic
}

\begin{abstract}
In this paper the performance of Multi-chaotic PSO algorithm is investigated using two shifted benchmark functions. The purpose of shifted benchmark functions is to simulate the time-variant real-world problems. The results of chaotic PSO are compared with canonical version of the algorithm. It is concluded that using the multi-chaotic approach can lead to better results in optimization of shifted functions.
\end{abstract}

Keywords: Particle swarm optimization, PSO, Chaos, Shifted functions, Multi-chaotic, Pseudo-random numbers

\section{INTRODUCTION}

In recent years there has been a significant development in the area of evolutionary computational techniques (ECTs) such as the PSO algorithm [1-4]. One of the promising approaches is the implementation of chaotic sequences as Pseudo-random number generators (PRNGs) [5 - 11]. In this research the performance of PSO algorithm with multi-chaotic PRNG [9] is investigated on two shifted benchmark functions. The shifted benchmark functions are designed in order to better simulate the time-variant real-world problems.

\section{PARTICLE SWARM OPTIMIZATION ALGORITHM}

The PSO algorithm is inspired in the natural swarm behavior of birds and fish. It was introduced by Eberhart and Kennedy in 1995 [1]. Each particle in the population represents a candidate solution for the optimization problem that is defined by the cost function (CF). In each iteration of the algorithm, a new location (combination of $\mathrm{CF}$ parameters) for the particle is calculated based on its previous location and velocity vector (velocity vector contains particle velocity for each dimension of the problem). Within this research the PSO algorithm with global topology (GPSO) [6] was utilized. The chaotic PRNG is used in the main GPSO formula (1), which determines a new "velocity", thus directly affects the position of each particle in the next iteration.

$$
v_{i j}^{t+1}=w \cdot v_{i j}^{t}+c_{1} \cdot \text { Rand }\left(\text { pBest }_{i j}-x_{i j}^{t}\right)+c_{2} \cdot \text { Rand }\left(g_{\text {Best }}-x_{i j}^{t}\right)
$$

Where:

$\mathrm{v}_{i}^{t+1}$ - New velocity of the $i t h$ particle in iteration $t+1$.

$w$ - Inertia weight value; $\mathrm{v}_{i}^{t}$ - Current velocity of the $i$ th particle in iteration $t ; c_{1}, c_{2}$ - Priority factors; $p B_{\text {Best }} t_{\mathrm{i}}-$

Personal best solution found by the $i t h$ particle; $g B e s t$ - Best solution found in a population; $\mathrm{x}_{i j}{ }^{t}$ - Current position of the $i$ th particle (component $j$ of dimension $D$ ) in iteration $t$; ; Rand - Pseudo random number, interval $(0,1)$. CPRNG is applied only here.

The maximum velocity was limited to 0.2 times the range as it is usual. The new position of each particle is then given by (2), where $x_{\mathrm{i}}^{\mathrm{t}+1}$ is the new particle position:

$$
x_{i}^{t+1}=x_{i}^{t}+v_{i}^{t+1}
$$

Finally the linear decreasing inertia weight $[3,4]$ is used in the typically referred GPSO design that was used in this study. The inertia weight has two control parameters wstart and wend. A new w for each iteration is given by (3), where $\mathrm{t}$ stands for current iteration number and $\mathrm{n}$ stands for the total number of iterations. The values used in this study were wstart $=0.9$ and wend $=0.4$.

$$
w=w_{\text {start }}-\frac{\left(\left(w_{\text {start }}-w_{\text {end }}\right) \cdot t\right)}{n}
$$




\section{CHAOTIC MAPS}

In this section two discrete chaotic systems that were used as CPRNGs are presented.

Lozi Map

The Lozi map is a simple discrete two-dimensional chaotic map. The map equations are given in (4). The parameters used in this work are: $a=1.7$ and $b=0.5$ with respect to [11]. For these values, the system exhibits typical chaotic behavior and with this parameter setting it is used in the most research papers and other literature sources.

$$
\begin{aligned}
& X_{n+1}=1-a\left|X_{n}\right|+b Y_{n} \\
& Y_{n+1}=X_{n}
\end{aligned}
$$

\section{Arnold's Cat Map}

The Arnold's Cat map is a simple two dimensional discrete system that stretches and folds points ( $\mathrm{x}, \mathrm{y})$ to $(\mathrm{x}+\mathrm{y}$, $\mathrm{x}+2 \mathrm{y}) \bmod 1$ in phase space. The map equations are given in Eq. 5. This map was used with parameter $\mathrm{k}=0.1$.

$$
\begin{aligned}
& X_{n+1}=X_{n}+Y_{n}(\bmod 1) \\
& Y_{n+1}=X_{n}+k Y_{n}(\bmod 1)
\end{aligned}
$$

\section{TEST FUNCTIONS}

In order to investigate on the performance of multi-chaotic PSO algorithm on functions closer to real problem than static test function, two shifted function were chosen. Shifted function global optimum moves with each start of the algorithm but keeps their basic characteristic thus simulates the time-variant real problems. Following shifted test functions were used in this study.

Shifted Sphere function is given by (6).

$$
f(x)=\sum_{i=1}^{\operatorname{dim}}\left(x_{i}-\text { shift }\right)^{2}
$$

Function minimum: Position for $\mathrm{E}_{\mathrm{n}}:\left(\mathrm{x}_{1}, \mathrm{x}_{2} \ldots \mathrm{x}_{\mathrm{n}}\right)=$ shift; Value for $\mathrm{E}_{\mathrm{n}}$ : $\mathrm{y}=0$; Shifted Rastrigin`s function is given by (7).

$$
f(x)=10 \operatorname{dim}+\sum_{i=1}^{\operatorname{dim}}\left(x_{i}-\text { shift }_{i}\right)^{2}-10 \cos \left(2 \pi x_{i}-\text { shift }_{i}\right)
$$

Function minimum: Position for $\mathrm{E}_{\mathrm{n}}:\left(\mathrm{x}_{1}, \mathrm{x}_{2} \ldots \mathrm{x}_{\mathrm{n}}\right)=$ shift; Value for $\mathrm{E}_{\mathrm{n}}$ : $\mathrm{y}=0$; Shift $_{i}$ is an random number from interval $<-5.11,5.11>$. Where $<-5.11,5.11>$ are the low and high bounds for the population individuals. Shift value is generated on the start of optimization process.

\section{EXPERIMENT}

The control parameters of PSO algorithm were set as follows:

Pop. size: 40; N. of iterations: 5000; $\mathrm{w}_{\text {start }}: 0.9 ; \mathrm{w}_{\text {end }}:$ 0.4; Dimension: 10; Runs: 50. Two different instances of multi-chaotic PSO [9] are investigated here. In the multi-chaotic approach two different CPRNGs are switched when the algorithm seems to stagnate (for details see [9]). In the first design in this study the optimization starts with Lozi map based CPRNG and it is switched to CPRNG based on Arnold's Cat map. In the second design the CPRNGs are used in opposite order. The results are summarized in Tables 1 and 2. Furthermore mean gBest history for each function is depicted in Fig. 1 and Fig. 2.

TABLE 1. Mean results comparison - Shifted Sphere function

\begin{tabular}{l|lll} 
& GPSO & Lozi - Arnold & Arnold - Lozi \\
\hline Sphere & 0.000289213 & 0.00183071 & $\mathbf{0 . 0 0 0 0 4 2 0 8 2}$ \\
Mean CF Value: & 0.00128856 & 0.012187 & 0.000171946 \\
Ctd. Dev.: & 0. & 0. & 0. \\
Max. CF Value: & 0.00863989 & 0.0870667 & 0.00121531 \\
Min. CF Value: & 0. & 0. & 0. \\
\hline
\end{tabular}




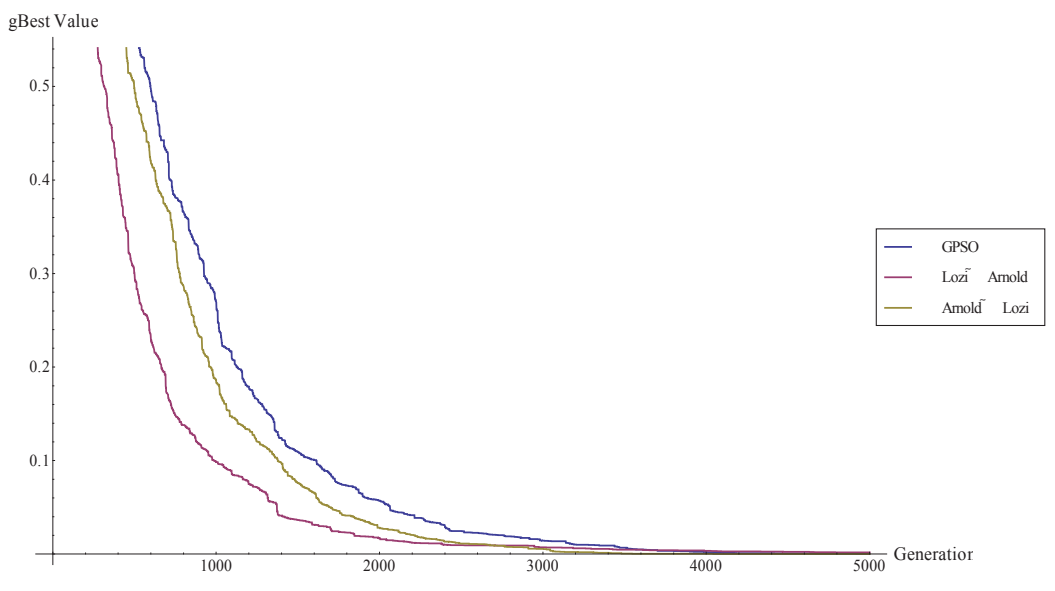

FIGURE 1. Mean gBest history for the Sphere function

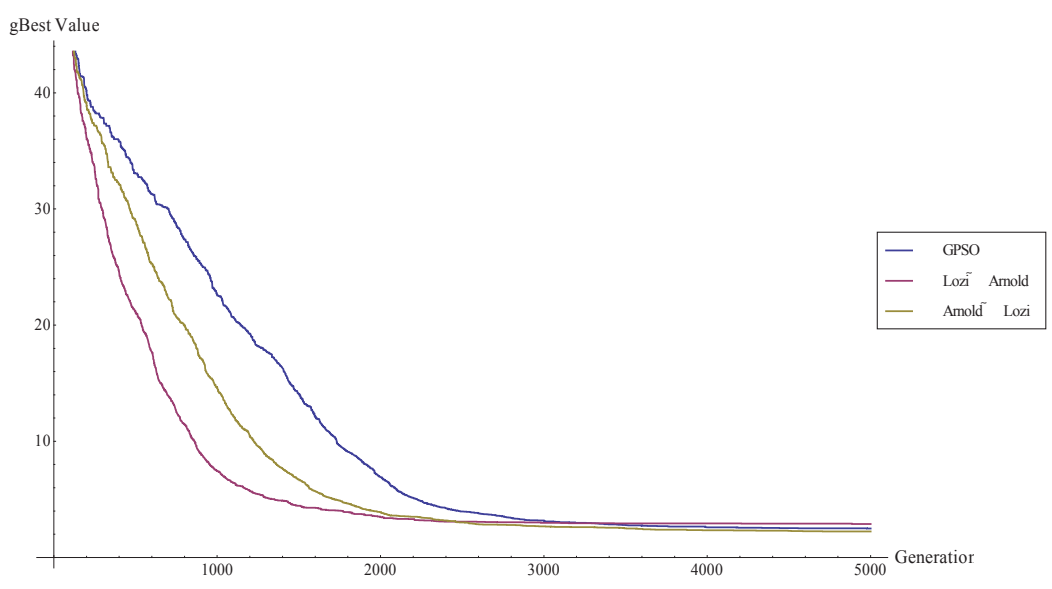

FIGURE 2. Mean gBest history for the Rastrigin's function 
TABLE 2. Mean results comparison - Shifted Rastrigin's function

\begin{tabular}{l|lll}
\hline Rastrigin & GPSO & Lozi - Arnold & Arnold - Lozi \\
\hline Mean CF Value: & 2.49478 & 2.88599 & $\mathbf{2 . 2 2 9 9 5}$ \\
Std. Dev.: & 1.53435 & 1.64208 & 1.52427 \\
CF Value Median: & 1.99001 & 2.98488 & 1.98992 \\
Max. CF Value: & 6.96471 & 6.96471 & 6.2411 \\
Min. CF Value: & 0. & 0. & 0. \\
\hline
\end{tabular}

\section{CONCLUSION}

In this study the performance of multi-chaotic PSO was investigated on two different shifted benchmark functions. The aim was to investigate the performance of this design on closer to real-world problems. Results presented in this work support claim that using two different CPRNGs within one run of the algorithm may improve the performance of PSO algorithm on various optimization tasks. The second designed combination of Arnold's Cat map based CPRNG and Lozi map based CPRNG outperformed the canonical version in both cases. This promising result should motivate future research of this approach.

\section{ACKNOWLEDGMENTS}

This work was supported by Grant Agency of the Czech Republic - GACR P103/13/08195S, Grant of SGS No. SP2014/159, VŠB - Technical University of Ostrava, Czech Republic, by the Development of human resources in research and development of latest soft computing methods and their application in practice project, reg. no. CZ.1.07/2.3.00/20.0072 funded by Operational Programme Education for Competitiveness, co-financed by ESF and state budget of the Czech Republic, by European Regional Development Fund under the project CEBIA-Tech No. CZ.1.05/2.1.00/03.0089 and by Internal Grant Agency of Tomas Bata University under the project No. IGA/FAI/2014/010.

\section{REFERENCES}

1. Kennedy, J., Eberhart, R.: Particle swarm optimization. In: IEEE International Conference on Neural Networks, 1995, pp. 1942-1948.

2. Kennedy, J., Eberhart, R.C., Shi, Y.: Swarm Intelligence. Morgan Kaufmann Publishers, (2001).

3. Nickabadi, A., Ebadzadeh, M.M., Safabakhsh, R.: A novel particle swarm optimization algorithm with adaptive inertia weight. Applied Soft Computing 11(4), 3658-3670 (2011).

4. Yuhui, S., Eberhart, R.: A modified particle swarm optimizer. In: IEEE World Congress on Computational Intelligence., 4-9 May 1998, pp. 69-73.

5. R. Caponetto, L. Fortuna, S. Fazzino, M.G. Xibilia, Chaotic sequences to improve the performance of evolutionary algorithms, Evolutionary Computation, IEEE Transactions on, vol.7, no.3, pp. 289- 304, June 2003

6. Araujo, E., Coelho, L., Particle swarm approaches using Lozi map chaotic sequences to fuzzy modelling of an experimental thermal-vacuum system, Applied Soft Computing, v.8 n.4, p.1354-1364, September, 2008

7. Alatas B., Akin E., Ozer B. A., Chaos embedded particle swarm optimization algorithms, Chaos, Solitons \& Fractals, Volume 40, Issue 4, 30 May 2009, Pages 1715-1734, ISSN 0960-0779

8. Pluhacek M., Senkerik R, Davendra D., Kominkova Oplatkova Z., and Zelinka I., "On the behavior and performance of chaos driven PSO algorithm with inertia weight," Computers \& Mathematics with Applications, vol. 66, pp. 122-134, 2013.

9. Pluhacek, M., Senkerik, R., Zelinka, I.: Particle swarm optimization algorithm driven by multichaotic number generator. Soft Comput 18(4), 631-639 (2014). doi:10.1007/s00500-014-1222-z

10. Pluhacek M., Senkerik R,, Davendra D., Zelinka I., Designing PID Controller For DC Motor System By Means Of Enhanced PSO Algorithm With Discrete Chaotic Lozi Map, In: Proceedings of the 26th European Conference on Modelling and Simulation, ECMS 2012, pp. 405 - 409, 2012, ISBN 978-0-9564944-4-3.

11. Sprott, J. C., "Chaos and Time-Series Analysis“, Oxford University Press, 2003 
AIP Conference Proceedings is copyrighted by AIP Publishing LLC (AIP). Reuse of AIP content is subject to the terms at: http://scitation.aip.org/termsconditions. For more information, see http://publishing.aip.org/authors/rights-and-permissions. 